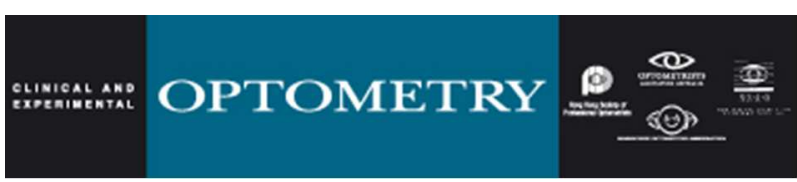

\title{
Reliability in perimetric multichannel contrast sensitivity measurements
}

\begin{tabular}{|r|l|}
\hline Journal: & Clinical and Experimental Optometry \\
\hline Manuscript ID: & CEOptom-13-282-OP.R1 \\
\hline Manuscript Type: & Original Research Paper \\
\hline Date Submitted by the Author: & n/a \\
\hline Complete List of Authors: & $\begin{array}{l}\text { de Fez, Dolores; Universidad de Alicante, D. Óptica, Farmacología y } \\
\text { Anatomía } \\
\text { Capilla, Pascual; Universidad de Valencia, D. Óptica } \\
\text { Camps, Vicente; Universidad de Alicante, D. Óptica, Farmacología y } \\
\text { Anatomía } \\
\text { Luque, Maria José; Universidad de Valencia, D. Optica } \\
\text { Moncho, Vicenta; Universidad de Alicante, D. Óptica, Farmacología y } \\
\text { Anatomía }\end{array}$ \\
\hline Keywords: & \begin{tabular}{l} 
repeatability, reproducibility, contrast sensitivity, multichannel perimetry \\
\hline
\end{tabular} \\
\hline
\end{tabular}

SCHOLARONE ${ }^{m}$

Manuscripts 
Title: Reliability in perimetric multichannel contrast sensitivity measurements Running title: Reliability in perimetric multichannel

\author{
Authors \\ Dolores de Fez (PhD), \\ Pascual Capilla ${ }^{+}(\mathrm{PhD})$, \\ Vicente Camps (PhD), \\ $M^{a}$ José Luque ${ }^{+}(P h D)$, \\ Vicenta Moncho (DOO) \\ Dpto. Óptica, Farmacología y Anatomía, Universidad de Alicante \\ + Dpto. Óptica, Universidad de Valencia
}

\title{
Corresponding Author
}

Dolores de Fez

Dpto. Óptica, Farmacología y Anatomía, Universidad de Alicante, Carretera San Vicente del Raspeig s/n - 03690 San Vicente del Raspeig Alicante 
Background: In this study, the reliability of perimetric contrast sensitivity measurements favouring the achromatic, the red-green and the blue-yellow postreceptorial mechanisms was analysed.

Methods: A new technique, multichannel ATD perimetry, provides spatial and temporal stimuli favouring the detection by an achromatic mechanism (A), from a magno or parvocellular origin, or by a red-green $(R G)$ chromatic mechanism, with a parvocellular origin, or a blue-yellow (BY) mechanism, with a koniocellular origin. The repeatability and reproducibility of contrast sensitivity measurements with these stimuli were studied in a group of 40 healthy subjects. The analysis was carried out on 21 testing points within a $60^{\circ} \times 40^{\circ}$ foveacentered region of the visual field.

Results: The within-observer repeatability for the four mechanisms studied is either good or excellent when the Intra-Class Correlation Coefficient (ICC) can be calculated. For the remaining points, the Friedman's test finds that the measurements are repeatable. The between-observer reproducibility was either excellent or good in cases where the ICC applied and according to the Friedman's test all results were reproducible.

Conclusions: The results obtained showed good repeatability and reproducibility with achromatic, red-green and blue-yellow stimuli, although with BY stimuli repeatability is slightly worse. Future studies on the diagnostic validity of this device, are based on the fact that changes of sensitivity can be compared by means of a visual single task, contrast sensitivity measurement, and using a common metric. 
KEYWORDS: multichannel perimetry, repeatability, reproducibility, agreement, contrast sensitivity

The diagnosis of alterations of the visual system at an early stage is one of the main objectives pursued by vision researchers. Early detection could stop the loss of visual capabilities- even if not at present, maybe in the future, as increasingly effective treatments are being developed- or at least minimize the negative effects that some treatments produce.

It has been shown that diseases affecting visual function can damage the mechanisms involved in spatial, chromatic and motion processing ${ }^{1}$, causing losses in contrast sensitivity both with stationary ${ }^{2}$ and moving ${ }^{3}$ stimuli, increasing thresholds in dark adaptation ${ }^{4}$ and provoking either generalized colour contrast sensitivity losses or specific losses along the blue-yellow or de red-green axes. Furthermore, congenital and acquired defects, caused by diseases, medications or unhealthy habits, influence colour perception and contrast sensitivity. For instance, losses in the chromatic or achromatic mechanisms, or in both, have been reported in glaucoma ${ }^{1,5-8}$, ocular hypertension $^{9}$, optical neuritis ${ }^{10}$, AMD (Age-related Macular Degeneration) ${ }^{11}$, diabetes $^{2-4,12,13}$, multiple sclerosis ${ }^{10}$, or Parkinson's disease ${ }^{14}$.

The perimetry tests more frequently used to diagnose and evaluate losses of visual function are SAP perimetry (measurement of luminance thresholds with white-on-white stimuli), SWAP (measurement of the luminance thresholds to detect a blue stimulus against a yellow background) and FDT (contrast thresholds with spatio-temporal achromatic stimuli). Both SWAP ${ }^{13}$, and 
$\mathrm{FDT}^{15}$ perimetry seem capable of detecting functional damage in different regions of the visual field before other characteristic symptoms of certain diseases appear. For example, detection of damage in 10 to $30 \%$ of hypertensive subjects ${ }^{16}$ and in $33.3 \%$ of subjects with suspected optic nerve damage with SWAP has been reported ${ }^{17}$. A more specific test for the parvocellular pathway, the high-resolution perimetry (HPRP), detects damage in 15 to $24 \%$ of hypertensive subjects ${ }^{16}$. Nowadays, to assess different visual mechanisms of a patient, it is necessary to use a combination of devices. For example, FTD perimetry to analyze the magnocellular pathway ${ }^{18,19}$, HPRP to analyze the parvocellular pathway ${ }^{20,21}$ or SWAP perimetry for the koniocellular pathway ${ }^{22,23}$. This identification of visual tasks with particular cellular pathways is perhaps too simplistic (we refer the interested reader to Kaplan ${ }^{24}$ ) and it is not always clear how well a given technique isolates the responses of a particular mechanism. See, for instance, White et al ${ }^{25}$ for a discussion about the relative contributions of the magno and parvo pathways in the detection of FDT stimuli. In the case of the blue-yellow mechanisms, although other cells with S-cone input might mediate an S-off pathway ${ }^{26,27}$, they represent a very small percentage of the total population of cells with S-cone input ${ }^{28}$, and their role in detection tasks and in colour appearance is not clear at present. But admitting that these different techniques do indeed favour different mechanisms, we find that neither the tasks carried out by the patient nor the metrics used to express the results are comparable, making the analysis of the relative losses incurred by each mechanism difficult. In addition, commercial devices limit the capability of the user to configure the spatial-temporal and chromatic characteristics of the 
stimuli to adapt the design to particular aims of study -although recent efforts have been made to overcome this limitation ${ }^{29}$. For these two reasons, it would be desirable to have a device with stimuli that could be configured to focus on different visual pathways. In order to improve the performance of existing diagnostic technologies, the Vision Group of the University of Valencia has developed a new multichannel contrast sensitivity perimetry technique named "ATD Multichannel Functional Test"30. A, T and D stand in different colour vision models for the three post-receptorial mechanisms, "achromatic" (A), "red-green" (because this is the colour mechanism left to Tritanopes, hence the T) and "blue-yellow" (because this is the colour mechanism left to Deuteranopes, hence the D). Since this notation is not usual in clinical research, we will use in what follows RG and BY instead when referring to the chromatic mechanisms. The examiner may define stimuli in the desired directions in colour space ${ }^{31,32}$ and choose the spatial and temporal frequency of the stimulus to favour a particular mechanism (for example, magno or parvo when using achromatic stimuli) while ensuring that the same task is performed by the patient for each stimulus modality (see Appendix). This feature is essential in the process of searching for the optimal stimulus to detect and evaluate damage caused by a given pathology. For instance, in a recent study we have shown that chromatic red-green and blue-yellow patterns with low spatial $(0.5 \mathrm{cpd})$ and temporal (2 $\mathrm{Hz}$ ) frequencies could be more sensitive for early detection of glaucomatous damage than achromatic patterns, including low spatial-high temporal frequency doubling stimuli (FDT perimetry) ${ }^{33,34}$. 
The aim of our study is to demonstrate the reliability of the psychophysical procedure used, assessing the repeatability and reproducibility of the measurements made with our technique/device. The precision of a device or clinical method is a factor that should be considered when conducting method comparison studies. If a device has poor precision it is unlikely to have good agreement with another device. Hence, comparing the precision of the two devices or methods will provide greater insight into the source of eventual differences. Repeatability and reproducibility are the two sides of precision ${ }^{35}$. Repeatability refers to the variability in repeated measurements by one subject when all other factors are assumed constant (within-observer variability). Reproducibility refers to the variability in repeated measurements when one or more factors, such as observer, instrument, calibration or time is varied (between-observer variability). In this paper, the changing factor is the clinician conducting the test.

\section{METHODOLOGY}

\section{Device}

The ATD multichannel perimetry provides spatial and temporal stimuli for determining contrast sensitivity measurements at different testing points in the visual field, using a staircase psychophysical method. To evaluate the achromatic mechanism $A$, a stimulus favouring the magnocellular pathway $(A-$ $0.5 \mathrm{cpd} / 12 \mathrm{~Hz})$ and other stimulus stimulating the parvocellular pathway (A$4 \mathrm{cpd} / 2 \mathrm{~Hz}$ ) were chosen. A large corpus of literature shows that the appearance of spatio-temporal patterns at detection threshold depends on the ratio between 
temporal and spatial frequency. Though frontiers are not sharply defined, observers perceive either the temporal or the spatial pattern of the stimulus at threshold, depending on whether the frequency ratio is either above 1-2 degrees/second or below this value ${ }^{36-40}$. According to the prevalent theory linking visual functions and visual pathways (see again $\mathrm{Kaplan}^{28}$ ), this result would imply that detections would be mediated either by the magno or by the parvo pathway, respectively. Our choice of stimuli is also supported by experiments on selective lesions, which show that damage in the parvo pathway greatly impairs the detectability of gratings in all the spatio-temporal domain, except for the high temporal-low spatial frequency corner, and the contrary happens when damage is confined to the magnocellular pathway ${ }^{41-43}$. It is not possible, however, to ensure that the parvo pathway does not contribute to the detection of the $\mathrm{A}-0.5 \mathrm{cpd} / 12 \mathrm{~Hz}$ stimuli (see Anton et al. ${ }^{34}$ for a discussion). The red-green and blue-yellow chromatic mechanisms, putatively mediated by the parvo and koniocellular pathways, respectively, were evaluated by stimuli modulated along the RG and BY directions of color space, with a spatial frequency of $0.5 \mathrm{cpd}$ and a temporal frequency of $2 \mathrm{~Hz}(\mathrm{RG}-0.5 \mathrm{cpd} / 2 \mathrm{~Hz}$ and BY-0.5cpd/2Hz). The choice of these stimuli fulfilled two conditions: first, the dynamic range of the device still allowed the measurement of the subject's threshold ${ }^{44}$ and second, the differences between normal and glaucomatous patients were the largest we had obtained with our device ${ }^{34}$. For a more detailed explanation of the stimuli used, see the Appendix and Anton et al. ${ }^{34}$.

\section{Measurement procedure}


Measurements were carried out in a darkened room. During a session, contrast sensitivity was measured at the 21 testing points described in the Appendix. The screen is placed at $25 \mathrm{~cm}$ from the patients and therefore a 4.00 D lens was added to their refraction to avoid accommodation. After an adaptation period of 30 seconds, the fixation stimulus flickered to signal the beginning of the test and the first trial was presented. The subject was instructed to press a button if any variation from the background was detected at any point of the visual field. Subjects' responses caused the stimulus to disappear from the display but counted as detections only if they occurred 100 ms after stimulus onset and before stimulus offset. The maximum duration of the stimulus was 1 second. The time interval between trials was randomized by the program, from 200 to $500 \mathrm{~ms}$, to minimize the likelihood that subjects would engage in rhythmic responses.

Thresholds were determined by an interleaved stepwise threshold algorithm. At each trial, the testing point was changed at random. In the first trial at a given testing point, the stimulus had the maximum amplitude achievable by the CRT. If this stimulus was detected, amplitude was divided by 2 at the next trial at that point, and continued decreasing in this way until the subject failed to detect the stimulus. The staircase was then reversed and amplitude increased by a $\sqrt{2}$ factor for the next presentation and continued increasing in this way until the test was again detected. This triggered a second reversal, and amplitude was divided by $\sqrt[4]{2}$, and so on. Thus, the amplitudes (see Appendix) at two consecutive trials at the same region, $\Delta R_{k-1}$ and $\Delta R_{k}$, relate to each other as follows (Equation 1): 
$\log _{2}\left(\Delta R_{k}\right)=\log _{2}\left(\Delta R_{k-1}\right)+\frac{(-1)^{n+1}}{2^{n}}$

where $n$ is the number of reversals until trial $k$. The criteria for exiting the staircase procedure at a given point were either totaling four reversals or 20 presentations, whatever came first. The staircase was also interrupted at a given point if a series of 5 consecutive stimuli with maximum amplitude passed undetected. Once a staircase was finished, threshold at that point, $\Delta R_{\text {thres }}$, was defined as the amplitude value of the last detected stimulus. If no stimulus was detected, threshold was defined as the maximum amplitude value achievable by the device in the corresponding cardinal direction. Contrast sensitivity in decibels (dB) was computed as (Equation 2):

$S=10 \log _{10} \frac{\Delta R_{\max }}{\Delta R_{\text {thres }}}$

where $\Delta R_{\max }$ is the maximum generable amplitude along the direction of the stimulus.

\section{Control stimuli}

Among the stimuli presentations, up to 16 false positive trials and 10 false negative trials were also randomly interleaved. Additionally, each session included up to 8 fixation-losses catch trials, presentations in the blind spot location previously estimated for the subject. These are $1.5^{\circ} \times 1.5^{\circ}$ squares with the same chromatic and spatial modulation as the false negative trials 
(achromatic with $f_{x}=2 \mathrm{cpd}$, without flicker), with maximum amplitude. Test results were rejected if either the false positive or the false negative rate was over $33 \%$, or if fixation losses surpassed $20 \%$.

\section{Selection of subjects}

We worked with a group of 40 healthy subjects aged between 20-35 and measures were taken in one eye randomly chosen. The ocular and medical history of the participants was examined, to discard those subjects with symptoms or familiar antecedents of visual or systemic diseases affecting vision. Preliminary tests included refraction, assessment of the anterior ocular segment with a Topcon SL8Z Biomicroscope and of the posterior segment with a Topcon TRC-NW6S Non-Mydriatic Retinal Camera, non-contact tonometry with AT900®-Haag-Streit and the Farnsworth-Munsell 100-Hue colour-test. Inclusion criteria were absence of ocular and systemic diseases that could affect vision, intra-ocular pressure (IOP) values below $21 \mathrm{~mm} \mathrm{Hg}$, spherical equivalent below 4D and cylinder below 2D, 20/25 Snellen visual acuity or better and normal chromatic discrimination as assessed by the FarnsworthMunsell100-Hue test. The study adheres to the tenets of the Declaration of Helsinki for Research Involving Human Observers.

\section{Experimental design and development of the measurement sessions}

The 40 subjects were divided into two groups of 20 subjects each. Group 1 underwent testing with stimulus $\mathrm{A}-0.5 \mathrm{cpd} / 12 \mathrm{~Hz}$ and $\mathrm{A}-4 \mathrm{cpd} / 2 \mathrm{~Hz}$, to evaluate, 
respectively, the achromatic mechanisms of magnocellular and parvocellular origin (in what follows, A-Magno and A-Parvo for short). Group 2 underwent testing for the red-green (RG-0.5cpd/2Hz) and blue-yellow (BY-0.5cpd/2Hz) chromatic mechanisms (in what follows RG and BY for short).

Each subject underwent four perimetry tests with each of the two stimuli assigned to their group: three perimetry tests were conducted by Clinician 1 and one by Clinician 2. Data measured by Clinician 1 was used in the repeatability study, the comparison between Clinicians 1 and 2 constituted the reproducibility study. Tests took place on different days to avoid the influence of fatigue. In the first day, the preliminary tests to determine whether the subject met the inclusion criteria were carried out and the subject performed the four perimetry tests for each of the two stimuli assigned to his/her group in two different days. The first two tests were conducted by Clinician 1 , in the two last ones Clinicians 1 and 2 alternated in random order. In this way, we expected to reduce possible learning effects in the reliability study.

Each perimetry test took about 4 to 8 minutes, depending on the subject and on the stimulus characteristics (A-Magno: 7.7 $\pm 0.7 \mathrm{~min}, \mathrm{~A}-\mathrm{Parvo:}$ 4.1 $\pm 0.6 \mathrm{~min}, \mathrm{RG}: 4.9 \pm 0.4 \mathrm{~min}, \mathrm{BY}: 5.7 \pm 0.4 \mathrm{~min})$. The subject rested for 10 minutes between perimetry tests and the duration of a complete measurement session was always kept below one hour.

\section{Statistical analysis}

The statistical tests were performed using SPSS v. 14.0.1 for Windows (SPSS Inc., Chicago, IL, USA). Besides analyzing the results obtained at the 
different testing points, we have considered groupings in three zones: the fovea (point 11, see Table 1), the perifovea, which comprises the four points surrounding the fovea (points 8, 9, 13 and 14) and the extrafovea (remaining points).

The study of the reliability of our device follows the guidelines laid out by the International Organization for Standardization (IOS) and we have adopted their definitions of repeatability and reproducibility ${ }^{35}$. In the literature on automated perimetry reliability with normal subjects, a great variety of methodologies is used, but the IOS guidelines are not followed ${ }^{45-47}$. The normality of the samples was evaluated using the Shapiro-Wilk test at the $95 \%$ significance level, as recommended for samples with less than 30 subjects, and the appropriate parametric or non-parametric tests applied in consequence ${ }^{48-50}$.

For normally distributed data, the concordance-coincidence between multiple measures of the same variable was assessed with the Intraclass Correlation Coefficient (ICC) $)^{51}$, both in the study of within-observer concordance (repeatability) and in the study of between-observer concordance (reproducibility). According to the value of this coefficient, measurement reliability is labelled as: absent $(0)$, low $(<0.4)$, between regular and good $([0.4-$ $0.75])$ and excellent $(>0.75)^{51}$.

Friedman's nonparametric test of k-related samples was carried out if the distributions were not normal. An asymptotic significance greater than 0.05 with this test indicates that there are not significant differences between the measurements, that are therefore considered repeatable or reproducible ${ }^{48,49,52}$. 


\section{RESULTS}

Mean sensitivity values for each stimulus are summarized at Tables 1 and 2. It can be seen that the A-Magno mechanism has the highest values of sensitivity for all points, followed by the BY mechanism. On the contrary, the AParvo mechanism has very low values of sensitivity, except in the fovea and the perifovea. The RG mechanism shows the highest sensitivity averages in the fovea and perifovea and some points of the extrafovea $(4,5,17,18)$, but the values are always lower than those of the A-Magno mechanism. Sensitivity determines the mean number of trials (MNT) needed to measure threshold, therefore MNT decreases with eccentricity and is in general greater for the AMagno and BY stimuli than for RG and A-Parvo. The limit of 20 presentations is only occasionally reached with certain subjects at random locations with the AMagno stimulus, hardly $5 \%$ of the total number of measurements.

From the measures of sensitivity in the four mechanisms (A-Magno, AParvo, RG and BY) the repeatability of the instrument and the concordance between results from the two clinicians were analysed.

\section{Within-observer Repeatability}

The results of the repetability analysis for our four stimuli are summarized in Table 1. There appears the mean and the standard deviation of the sensitivity, and either the ICC values or the p-value of Friedman's test, as appropriate. Figure 1 presents the point-by-point repeatability classification for 
each of the four mechanisms. In this figure, the visual field has been divided in $10^{\circ} \times 10^{\circ}$ regions, centered in each of the testing points.

\section{Insert Figure 1 here}

In the A-Parvo mechanism, sensitivity data at most points at the extrafovea (76\%) present non-normal distributions. All these measurements are repeatable according to the Friedman's test. Fovea and perifovea present normal distributions and the repeatability is excellent in all cases (see ICC value). In the A-Magno mechanism, most of the points present normal distributions with repeatability between excellent (67\%) and good (19\%). At the perifovea, which does not follow the normal distribution (14\%), within-observer measurements are repeatable. In the RG mechanism, most points present a normal distribution and the repeatability is excellent $(62 \%)$, with a reduced number that are rated as just good (14\%). In all cases where the distribution is not normal $(24 \%)$, the Friedman's test proves that the measures are repeatable. In the BY mechanism most data distributions are normal, and the ICC values show that repeatability is excellent $(38 \%)$ or good $(48 \%)$. For points with nonnormally distributed data (14\%), the results are repeatable in all cases.

In summary, within-observer repeatability for the four mechanisms studied is either good or excellent when the ICC can be calculated. For the rest of points, the Friedman's test finds that the measurements are repeatable.

\section{Between-observer Reproducibility}

To check the reproducibility, we compared two measurements supervised by different clinicians. The third perimetry test of Clinician 1 was 
compared with the single measurerement performed by Clinician 2. As in the previous study, the normality of the sensitivity distributions was analysed and the appropriate reliability test for each case was applied. The results are shown in Table 2, using the same criteria as in Table 1. In Figure 2 presents the pointby-point reproducibility classification of for each of the four mechanisms.

\section{Insert Figure 2 here}

In the A-Parvo mechanism, most points in the extrafovea follow nonnormal distributions, and all points have proved to be reproducible according to the Friedman's test. As in the previous section, the distributions are normal in fovea and perifovea and reproducibility is excellent at all testing points. In most testing points, data from the A-Magno mechanism follow normal distributions with reproducibility between excellent (48\%) and good (19\%). In comparison with the data presented in the within-observer study, a larger number of extrafoveal points but just one at perifovea (up to a total of $33 \%$ ) do not follow the normal distribution. Friedman's analysis indicates that all the measurements are reproducible. With the RG stimulus, most points follow the normal distribution and reproducibility results are either excellent (52\%) or good (24\%). In all cases where the distribution is not normal (24\%), the Friedman's test indicates that the measurements are reproducible. With regard to the BY stimulus, most of the data distributions are normal, and the ICC test results are either excellent $(38 \%)$ or good $(48 \%)$. In the regions with non-normal distributions $(24 \%)$, the result is always reproducible. 
In summary, the between-observer reproducibility was either excellent or good in cases where the ICC applied and according to the Friedman test all results were reproducible.

\section{DISCUSSION}

A study of the reliability of the multichannel perimeter has been designed for the A-Magno, A-Parvo, RG and BY mechanisms. A sample of 40 normal subjects divided in two groups have participated in this study. All the subjects carried out four repeated perimetry tests, three under the supervision of a clinician (within-observer study) and one under the supervision of a different clinician (between-observer study). We have found that the within-observer measures are repeatable, i.e. there is no significant variability in the repetition of the measurements of an subject when other factors remain constant. In the between-observer study we have concluded that the measurements conducted by both clinicians are interchangeable.

In general, almost all measurements follow a normal distribution of the responses for all mechanisms. The points where sensitivity data are not normally distributed do not seem to follow a systematic pattern, common to all the stimuli, such as a dependence on eccentricity. The sole exception is the AParvo stimulus, which does not follow the normal distribution in the majority of the points, possible due to the subjects's low sensitivity outside the perifovea. However, in the fovea and the perifovea the distribution of responses follows the normal distribution. 
It is difficult to compare our reliability study with the literature, due to differences in the reliability criteria and in the number of measurements, in the structure of a measurement session and in the factors that change between measurements ${ }^{45-47}$. In spite of this, we may conclude that our multichannel perimeter presents standard deviation values similar to other studies with different devices and furthermore, our precision (ICC) results are as good as those obtained in other types of perimeter ${ }^{46,47}$.

The reasons for this good performance may lie in the psychophysical measurement procedure used and in the comparatively reduced number of points tested in the visual field. It has been shown that measurement repeatability depends on a large number of factors: number of testing points in the visual field and number of stimulus presentations in the measurement procedure $^{45,47,53,54}$, the spatio-temporal characteristics of the stimulus ${ }^{45,53,54}$, the patient's sensitivity (determined either by eccentricity ${ }^{53}$, age $\mathrm{e}^{47,53}$ or by damage in the visual system ${ }^{46}$ ), previous experience ${ }^{53,55}$, and so on... The four tests we have analysed have in common the distribution of testing points, the task to be performed by the observer and the psychophysical method, and therefore potential differences in repeatability must arise from the stimulus characteristics -which determine sensitivity, for instance, and therefore the number of trials needed to determine threshold, another relevant factor- or from the different limitations that the dynamic range of the device sets in each direction of colour space. The analysis of our results becomes complicated by the fact that there is not a common metric for repeatability for all testing points, and whereas ICC grades the results, the Friedman test doesn't. Considering only those testing 
points where the ICC could be computed, we used a general linear model to determine which variables determined repeatability and reproducibility. ICC was the dependent variable, mean sensitivity of the population sample, eccentricity, and mean number of trials needed to determine threshold -which changed with location in the visual field and stimulus type- were the independent variables and stimulus type was a factor. The analysis showed that the only significant difference was with stimulus type $(\sigma<0.001)$ and that repeatability results were significantly worse for the blue-yellow stimulus. Comparisons between SAP and SWAP perimetry also show that repeatability with blue-yellow stimuli is worse ${ }^{53}$. In the between-observer study, ICC did not significantly depend on any of the variables listed above.

We have shown, therefore, that the accuracy of the device, in general, is good, although it must still be shown that the same good results hold with an older population sample. The study with older adults is necessary and is at present a work on progress. Data from glaucoma and OHT subjects that we have previously published ${ }^{33}$ suggest that the $\mathrm{A}-4 \mathrm{cpd} / 2 \mathrm{~Hz}$ stimulus is likely to be the least useful of the four we have studied in this paper. The potential great advantage of this device is the versatility in designing visual stimulus, which allows a variety of studies based on the cells/mechanisms involved in the detection, which could help to find optimal stimuli for detecting and monitoring visual damage. For instance, it has been recently shown that chromatic redgreen and blue-yellow patterns with low spatial $(0.5 \mathrm{cpd})$ and temporal $(2 \mathrm{~Hz})$ frequencies could be more sensitive for early detection of a glaucomatous damage than achromatic patterns, including low spatial-high temporal frequency 
doubling stimuli (FDT perimetry) $)^{34}$. Moreover, these stimuli detected damage in ocular hypertensive and glaucoma suspect patients ${ }^{33,34}$. These results are promising for future use of the device for the early detection of pathologies that affect the visual system, when the relevant normative database of the normal population has been completed.

Disclosure of potential conflict of interest: Dolores de Fez, Vicente Camps and Vicenta Moncho have no proprietary interest in any material or instrument used in this study. P Capilla and MJ Luque, have an intellectual proprietary interest in the ATD multichannel perimeter patent (US 7.641.344 B2).

Acknowledgements. The ATD multichannel perimeter was built thanks to the support of the Spanish Ministerio de Ciencia y Tecnología Grants DPI2000-0116P4-02 and PTR 1995-0909-OP, in collaboration with INDUSTRIAS DE OPTICA SA (San Cugat del Vallés, Spain). 
Table 1: Results from the within-observer study. Mean sensitivities (dB) obtained in three measurements with Clinician 1, with their standard deviation (SD) and the results of the repeatability test (Friedman's p-value or ICC) in each mechanism, at each of the 21 testing points in the visual field. $(x, y)$ are the spatial coordinates of each point, in degrees, referred to a coordinate system with origin in fovea.

\begin{tabular}{|c|c|c|c|c|c|c|c|c|c|c|c|c|c|}
\hline \multirow[b]{2}{*}{ Point } & \multirow[b]{2}{*}{$(x, y)$} & \multicolumn{3}{|c|}{ A-Parvo } & \multicolumn{3}{|c|}{ A-Magno } & \multicolumn{3}{|c|}{ RG } & \multicolumn{3}{|c|}{ BY } \\
\hline & & mean $\pm \mathrm{SD}(\mathrm{dB})$ & FRIEDMAN & ICC & $\begin{array}{c}\text { mean } \pm S D \\
(\mathrm{~dB})\end{array}$ & FRIEDMAN & ICC & $\begin{array}{c}\text { mean } \pm S D \\
(\mathrm{~dB})\end{array}$ & FRIEDMAN & ICC & $\begin{array}{c}\text { mean } \pm S D \\
(\mathrm{~dB})\end{array}$ & FRIEDMAN & ICC \\
\hline 2 & $(-25,-5)$ & $0.15 \pm 0.54$ & 0.06 & & $11.2 \pm 3.4$ & & 0.94 & $0.4 \pm 1$ & 0.83 & & $4.2 \pm 1.9$ & & 0.86 \\
\hline 3 & $(-15,15)$ & $0.1 \pm 0.3$ & 0.06 & & $13.1 \pm 1.8$ & & 0.83 & $0.4 \pm 0.7$ & 0.35 & & $5.6 \pm 1.8$ & & 0.79 \\
\hline 4 & $(-15,5)$ & $1.3 \pm 1.4$ & 0.51 & & $14.2 \pm 1.4$ & & 0.9 & $4.6 \pm 1.4$ & & 0.86 & $8.3 \pm 1.2$ & & 0.63 \\
\hline 5 & $(-15,-5)$ & $1.2 \pm 1.5$ & 0.1 & & $15.3 \pm 1.6$ & & 0.62 & $4.8 \pm 1.3$ & & 0.9 & $8.4 \pm 1.8$ & & 0.71 \\
\hline 6 & $(-15,-15)$ & $0.2 \pm 0.7$ & 0.9 & & $12.6 \pm 3.3$ & & 0.93 & $1 \pm 1$ & & 0.9 & $6 \pm 1.6$ & & 0.7 \\
\hline 7 & $(-5,15)$ & $1.8 \pm 2.3$ & 0.62 & & $14.1 \pm 1.8$ & & 0.77 & $2.2 \pm 1.3$ & & 0.88 & $7 \pm 1.8$ & & 0.48 \\
\hline 8 & $(-5,5)$ & $5.3 \pm 3.6$ & & 0,95 & $16.4 \pm 2.4$ & 0.25 & & $8 \pm 1.6$ & & 0.8 & $8.7 \pm 1.8$ & 0.08 & \\
\hline 9 & $(-5,-5)$ & $4.6 \pm 3.3$ & & 0,92 & $16.3 \pm 2$ & 0.41 & & $7.9 \pm 1.4$ & & 0.8 & $8.8 \pm 1.7$ & & 0.6 \\
\hline 10 & $(-5,-15)$ & $1.3 \pm 1.5$ & 0.6 & & $14 \pm 2.7$ & & 0.92 & $3.5 \pm 1.2$ & & 0.85 & $7.2 \pm 1.8$ & & 0.56 \\
\hline 11 & $(0,0)$ & $8.3 \pm 3.5$ & & 0,9 & $18 \pm 2.4$ & & 0.55 & $10.8 \pm 2.2$ & 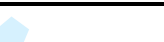 & 0.71 & $9.6 \pm 1.7$ & & 0.76 \\
\hline 12 & $(5,15)$ & $1.2 \pm 1.9$ & 0.07 & & $13.4 \pm 2$ & & 0.77 & $1.9 \pm 1.4$ & 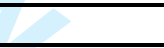 & 0.84 & $5.9 \pm 1.9$ & & 0.68 \\
\hline 13 & $(5,5)$ & $5.2 \pm 3$ & & 0,89 & $16.8 \pm 2.1$ & 0.35 & & $8.3 \pm 1.7$ & & 0.54 & $9 \pm 1.6$ & & 0.65 \\
\hline 14 & $(5,-5)$ & $5.3 \pm 3.1$ & & 0,81 & $16.9 \pm 1.2$ & & 0.69 & $8.3 \pm 1.5$ & & 0.64 & $9.1 \pm 1.8$ & 0.82 & \\
\hline 16 & $(15,15)$ & $0.1 \pm 0.5$ & 0.46 & & $13.6 \pm 1.3$ & & 0.87 & $0.7 \pm 0.8$ & 0.21 & & $5.4 \pm 1.6$ & & 0.8 \\
\hline 17 & $(15,5)$ & $1.1 \pm 1.6$ & 0.25 & & $14.6 \pm 1.4$ & & 0.81 & $4.2 \pm 1.5$ & & 0.82 & $6.7 \pm 1.7$ & & 0.72 \\
\hline 18 & $(15,-5)$ & $1.4 \pm 2$ & 0.26 & & $14.9 \pm 2.1$ & & 0.59 & $4.8 \pm 1.4$ & & 0.81 & $7.3 \pm 1.9$ & & 0.71 \\
\hline 19 & $(15,-15)$ & $0.5 \pm 1$ & 0.26 & & $13.8 \pm 3.5$ & & 0.97 & $2.7 \pm 1.3$ & & 0.84 & $6 \pm 2$ & & 0.79 \\
\hline 20 & \begin{tabular}{|l|}
$(25,5)$ \\
\end{tabular} & $0.2 \pm 0.4$ & 0.05 & & $13.2 \pm 3$ & & 0.88 & $1.8 \pm 1.4$ & & 0.83 & $5.2 \pm 2.6$ & & 0.84 \\
\hline 21 & $(25,-5)$ & $0.2 \pm 0.5$ & 0.33 & & $12.5 \pm 3.4$ & & 0.97 & $2 \pm 1.5$ & & 0.77 & $4.8 \pm 2.5$ & & 0.93 \\
\hline
\end{tabular}


Table 1: Results of the between-observer study. Mean sensitivities (dB) obtained in two measurements with two different clinicians, their standard deviation (SD) and the results of the reproducibility test (Friedman's p-value or ICC) in each mechanism, at each of the 21 testing points in the visual field. $(x, y)$ are the spatial coordinates of each point, in degrees, referred to a coordinate system with origin in fovea.

\begin{tabular}{|c|c|c|c|c|c|c|c|c|c|c|c|c|c|}
\hline \multirow[b]{2}{*}{ Point } & \multirow[b]{2}{*}{$(x, y)$} & \multicolumn{3}{|c|}{ A-Parvo } & \multicolumn{3}{|c|}{ A-Magno } & \multicolumn{3}{|c|}{$R G$} & \multicolumn{3}{|c|}{ BY } \\
\hline & & $\begin{array}{l}\text { mean } \pm S D \\
(\mathrm{~dB})\end{array}$ & FRIEDMAN & ICC & $\begin{array}{c}\text { mean } \pm S D \\
(\mathrm{~dB})\end{array}$ & FRIEDMAN & ICC & $\begin{array}{c}\text { mean } \pm S D \\
(\mathrm{~dB})\end{array}$ & FRIEDMAN & ICC & $\begin{array}{c}\operatorname{mean} \pm S D \\
(\mathrm{~dB})\end{array}$ & FRIEDMAN & ICC \\
\hline 2 & $(-25,-5)$ & $0.2 \pm 0.6$ & 0.18 & & $11 \pm 3.3$ & 0.25 & & $0.4 \pm 0.9$ & 0.65 & & $4.1 \pm 1.8$ & & 0.75 \\
\hline 3 & $(-15,15)$ & $0.05 \pm 0.2$ & 0.56 & & $13.2 \pm 2$ & 0.37 & & $0.4 \pm 0.7$ & 1 & & $5.6 \pm 1.2$ & & 0.77 \\
\hline 4 & $(-15,5)$ & $1.2 \pm 1.3$ & 1 & & $15.4 \pm 1.5$ & & 0.75 & $4.7 \pm 1.3$ & & 0.8 & $8.2 \pm 1.1$ & & 0.78 \\
\hline 5 & $(-15,-5)$ & $1 \pm 1.4$ & 0.8 & & $15.2 \pm 1.7$ & & 0.6 & $4.9 \pm 1.3$ & & 0.8 & $8.3 \pm 1.4$ & & 0.54 \\
\hline 7 & $(-5,15)$ & $1.8 \pm 2.1$ & 1 & & $14.4 \pm 1.7$ & & 0.75 & $2.3 \pm 1.4$ & & 0.8 & $7.1 \pm 1.6$ & & 0.63 \\
\hline 8 & $(-5,5)$ & $5 \pm 3.6$ & & 0.75 & $16.3 \pm 2.8$ & & 0.77 & $8 \pm 1.8$ & & 0.6 & $8.5 \pm 2$ & & 0.66 \\
\hline 9 & $(-5,-5)$ & $4.2 \pm 3.3$ & & 0.9 & $16.1 \pm 2.3$ & & 0.8 & $7.9 \pm 1.4$ & & 0.7 & $8.7 \pm 1.7$ & & 0.7 \\
\hline 10 & $(-5,-15)$ & $1.1 \pm 1.6$ & 1 & & $14.1 \pm 3$ & & 0.78 & $3.4 \pm 1.4$ & & 0.9 & $7.1 \pm 1.8$ & & 0.68 \\
\hline 11 & $(0,0)$ & $8 \pm 3.4$ & & 0.75 & $18 \pm 2.7$ & & 0.85 & $10.6 \pm 2.3$ & - & 0.8 & $9.6 \pm 1.7$ & & 0.85 \\
\hline 12 & $(5,15)$ & $1 \pm 1.7$ & 0.29 & & $13.4 \pm 2.3$ & & 0.5 & $2 \pm 1 . .5$ & 7 & 0.8 & $5.8 \pm 1.9$ & & 0.7 \\
\hline 13 & $(5,5)$ & $4.9 \pm 2.9$ & & 0.9 & $16.6 \pm 2.3$ & 0.25 & & $8.3 \pm 1.8$ & & 0.8 & $8.9 \pm 1.7$ & & 0.6 \\
\hline 14 & $(5,-5)$ & $4.9 \pm 3$ & & 0.75 & $16.8 \pm 1.2$ & & 0.7 & $8.1 \pm 1.6$ & & 0.6 & $9 \pm 1.8$ & & 0.7 \\
\hline 16 & $(15,15)$ & $0.1 \pm 0.3$ & 0.66 & & $13.5 \pm 1.3$ & & 0.78 & $0.7 \pm 0.9$ & 0.13 & & $5.3 \pm 1.4$ & 0.63 & \\
\hline 17 & $(15,5)$ & $1 \pm 1.5$ & 0.56 & & $14.4 \pm 1.5$ & & 0.6 & $4.3 \pm 1.5$ & & 0.9 & $6.3 \pm 1.7$ & & 0.78 \\
\hline 18 & $(15,-5)$ & $1.4 \pm 1.9$ & 0.62 & & $14.7 \pm 2.5$ & & 0.85 & $4.8 \pm 1.4$ & & 0.9 & $7.1 \pm 2.1$ & & 0.8 \\
\hline 19 & $(15,-15)$ & $0.5 \pm 1$ & 0.32 & & $13.8 \pm 3.5$ & 0.79 & & $2.7 \pm 1.4$ & & 0.9 & $6 \pm 2$ & 0.43 & \\
\hline 20 & \begin{tabular}{|l|}
$(25,5)$ \\
\end{tabular} & $0.1 \pm 0.3$ & 0.56 & & $13 \pm 3$ & & 0.81 & $2 \pm 1.4$ & & 0.84 & $2.5 \pm 2.5$ & & 0.95 \\
\hline 21 & $(25,-5)$ & $0.2 \pm 0.4$ & 0.41 & & $12.4 \pm 3.5$ & 0.65 & & $2.1 \pm 1.6$ & & 0.6 & $4.5 \pm 2.4$ & & 0.95 \\
\hline
\end{tabular}




\section{REFERENCES}

1. Pacheco-Cutillas M, Sahraie A, Edgar D. Acquired colour vision defects in glaucoma- their detection and clinical significance. British Journal of Ophthalmology. 1999;83:1396-402.

2. Verroti A, Lobefalo L, Petitti M, Mastropasqua L, Morgese G, Chiarelli F, et al. Relatioship between contrast sensitivity and metabolic control in diabetics with and without retinopathy. Ann Med. 1998;30:369-74.

3. Kawasaki K, Yonemura K, Yokogawa Y, Saito N, Kawakita S. Correlation between ERG oscillatory potential and psychophysical contrast sensitivity in diabetes. Doc Ophtalmol. 1986;64:209-15.

4. Greenstein V, Shapiro A, Hood D, Zaidi Q. Chromatic and luminance sensitivity in diabetes and glaucoma. J Opt Soc Am 1993;10:1785-91.

5. Leeprechanon N, Giangiacomo A, Fontana H, Hoffman D, Capriloli J. Frequency-Doubling Perimetry: Comparison with Standard Automated Perimetry to detect Glaucoma. American Journal of Ophtalmology. 2007;143:263-71.

6. Castelo-Branco M, Faria P, Forjaz V, Kozak LR, Azevedo H. Simultaneous comparison of relative damage to chromatic pathways in ocular hypertension and glaucoma: correlation with clinical measures. Invest. Ophthalmol. Vis. Sci. 2004;45:499-505.

7. McKendrick AM, Sampson GP, Walland MJ, Badcock DR. Contrast sensitivity changes due to glaucoma and normal aging: low-spatial-frequency 
losses in both magnocellular and parvocellular pathways. Invest. Ophthalmol. Vis. Sci. 2007;48:2115-2122.

8. Ferreras A, Polo V, Larrosa JM, et al. Can frequency-doubling technology and short-wavelength automated perimetries detect visual field defects before standard automated perimetry in patients with preperimetric glaucoma? J Glaucoma 2007;16:372-83.

9. Johnson CA, Brandt JD, Khong AM, Adams AJ. Short-wavelength automated perimetry in low-, medium-, and high-risk ocular hypertensive eyes. Initial baseline results. Arch Ophthalmol 1995;113:70-6.

10. Porciatti V, Sartucci F. Retinal and cortical evoked responses to chromatic contrast stimuli. Specific losses in both eyes of patients with multiple sclerosis and unilateral optic neuritis. Brain 1996;119(Pt 3):723-40.

11. Phipps J, Dang T, Vingrys A, Guymer R. Flicker perimetry losses in Agerelated Macular Degeneration. Invest. Ophthalmol. Vis. Sci. 2004;45(9):335560.

12. Nomura R, Terasaki $H$, Hirose $H$, Miyake $Y$. Blue-on-yellow perimetry to evaluate S cone sensitivity in diabetics. Ophthalmic Res. 2000;32:69-72. 13. Afrashi F, Erakgun T, Kose S, et al. Blue-on-yellow perimetry versus achromatic perimetry in type 1 diabetes without retinopathy. Diabetes Res Clin Pract 2003;61:7-11.

14. Müller T, Woitalla D, Peters S, Kohla k, Przuntek H. Progress of visual dysfunction in Parkinson's disease. Acta Neural Scand. 2002;105:256-60. 15. Realini T, Lai M, Barber, editors. Impact of diabetes on glaucoma screening using frequency-doubling perimetry. Conference Information: Annual Meeting of 
the Association-for-Research-in Vision-and-Ophtalmology, May 04-09; 2003; Ft Lauderdale, Fl: Ophtalmology.

16. Johnson C, Brandt J, Khong A, Adams A. Short-wavelength automated perimetry in low-, medium-, and high-risk ocular hypertensive eyes. Initial baseline results. Arc Ophthalmol. 1995;113:70-6.

17. Ferreras A, Polo V, Larrosa J. Can frequency-doubling technology and short-wavelength automated perimetries detect visual field defects before standard automated perimetry in subjects with preperimetric glaucoma? J Glaucoma. 2007;16:372-83.

18. Quigley H. Identification of glaucoma-related visual field abnormality with screening protocol of frequency doubling technology. Am J Ophthalmol. 1998;125:819-29.

19. Johnson C, Cioffi G, Van Buskirk E. Frequency doubling technology perimetry using a 24-2 stimulus presentation pattern. Optom and Vis Sci. 1999;76:571-81.

20. Wanger P, Oersson H. Pattern-reversal electroretinograms and high-pass resolution perimetry in suspected or early glaucoma. Ophtalmology. 1987;94:1098-103.

21. Chauhan B, LeBlanc R, McCornick T, Rogers J. Comparison high-pass resolution perimetry and pattern discrimination perimetry to conventional perimetry in glaucoma. Can J Ophtalmol. 1993;28:306-11.

22. Sample P, Johnson C, Haergerstrom-Portnoy G. Optimun parameters for short-wavelength automated perimetry. J Glaucoma. 1996;5:375-83. 
23. Sample P, Boswoth C, Weinreb R. Short-wavelength automated perimetry and motion automated perimetry in subjects with glaucoma. Arch Ophtalmol. 1997;115:1129-33.

24. Kaplan, E. (2008). The M, K, and P Streams in the Primate Visual System: what do they do for vision? Chapter 1.16, pages 369-382. The Senses. Elsevier, UK

25. White AJ, Sun $H$, Swanson WH, Lee BB. An examination of physiological mechanisms underlying the frequency-doubling illusion. Invest Ophthalmol Vis Sci. 2002;43:3590-3599.

26. Klug K, Herr S, Tratt Ngo I, Sterling P, Schen S. Macaque Retina Contains and S-cone off midget pathway. J Neurosci. 2003;23:9881-9887.

27. Dacey DM, Liao HW, Peterson BB, Robinson FR, Smith VC Pokorny J, King-Wai Y and Gamlin P. Melanopsin-expressing ganglion cells in primate retina signal colour and irradiance and project to the LGN. Nature, 2005, 433: 749-754.

28. Valberg A, Lee BB, Tigwell DA. Neurons with strong inhibitory S-cone inputs in the macaque lateral geniculate nucleus. Vision Res. 1986, 26:1061-1064.

29. Turpin A, Artes PH, McKendrick AM, The Open Perimetry Interface: an enabling tool for clinical visual psychophysics, Journal of Vision, 2012, 12, 1-5. 30. ATD Double Modulation Analyzer, patent US 7.641.344 B2 and 2246174 ES.

31. Krauskopf J WD, Heeley DW. Cardinal directions of colour space. Vision Res. 1982;22:1123-31. 
32. Derrington AM, Krauskopf J, Lennie P. Chromatic mechanisms in lateral geniculate nucleus of macaque. The Journal of Physiology. 1984;357:241-65. Epub 1984/12/01.

33. Morilla-Grasa A AA, Santamaría S, et al. Contrast sensitivity differences between glaucoma, ocular hypertensive and glaucoma suspect subjects found by ATD perimetry. Invest Ophtalmol Vis Sci ARVO. 2009;50(5):E-Abstract 5290. 34. Antón A, Capilla P, Morilla-Grasa A, Luque MJ, Artigas JM, FelipeA. Multichannel Functional Testing in Normal Subjects, Glaucoma Suspects, and Glaucoma Patients. Invest. Ophthalmol. Vis. Sci. December 2012 53:83868395; published ahead of print October 11, 2012, doi:10.1167/iovs.12-9944. 35. ISO. Accuracy (Trueness and Precision) of Measurement Methods and Results. Part1 and 2. Basic Methods for the Determination of Repeatability and Reproducibility of a Standard Measurement Method. In: Standardization. IOf, editor. Geneva, Switzerland: ISO, (ISO 5725-2); 1994.

36. Koenderink JJ, van Doorn AJ. Spatiotemporal contrast surface is bimodal. Opt Lett. 1979;4:32-34.

37. Harris MG. Velocity specificity of the flicker to pattern sensitivity ratio in human vision. Vision Res. 1980;20:687-691.

38. Burbeck CA. Criterion-free pattern and flicker thresholds. J Opt Soc Am. $1981 ; 71: 1343-1350$.

39. Murray I, MacCana F, Kulikowski JJ (1983). Contribution of two movement detecting mechanisms to central and peripheral vision. Vision Res. 1983;23: 151-159. 
40. Panish SC, Swift DJ, Smith RA (1983) Two-criterion threshold techniques: evidence for separate spatial and temporal mechanisms? Vision Res. 1983;23: 1519-1525.

41. Merigan WH, Katz LM y Maunsell JHR. The effects of parvocellular lateral geniculate lesions on the acuity and contrast sensitivity of macaque monkeys. J. Neurosci. 1991;11:994-1001.

42. Merigan W.H., Byrne C y Maunsell J.H.R. Does primate motion perception depend on the magnocellular pathway? J. Neurosci. 1991;11:3422-3429.

43. Merigan WH, Maunsell JHR. How parallel are the primate visual pathways? Ann Rev Neurosci. 1993;16:568-78.

44. Díez-Ajenjo M, Capilla P, Luque M. Red-green vs. blue-yellow spatiotemporal contrast sensitivity across the visual field. J Modern Optics. 2011;58:1736-1748.

45. Wall M, Woodward KR, Doyle CK, Artes PH. Repeatability of Automated Perimetry: A Comparison between Standard Automated Perimetry with Stimulus Size III and V, Matrix, and Motion Perimetry. Invest. Ophthalmol. Vis. Sci. 2009;50(2):974-9.

46. Kim LS, McAnany JJ, Alexander KR, Fishman GA. Intersession repeatability of Humphrey perimetry measurements in subjects with retinitis pigmentosa. Invest. Ophthalmol. Vis. Sci. 2007;48(10):4720-4.

47. Bengtsson B, Heijl A. Normal intersubject threshold variability and normal limits of the SITA SWAP and full threshold SWAP perimetric programs. Invest. Ophthalmol. Vis. Sci. 2003;44(11):5029-34. 
48. Hanneman S. Design, analysis and interpretation of method-comparison studies. AACN Avanced Critical Care. 2008;19:223-34.

49. Piñero D, Saenz-González C, Alió J. Intraobserver and interobserver repeatability of curvature and aberrometric measurements of the posterior corneal surface in normal eyes using Schiempflug photography. J Cataract Refract Surg. 2009;35(1):113-20.

50. Armstrong R, Davies L, Dunne M, Gilmartin B. Statistical guidelines for clinical studies of human vision. Ophthal. Physiol. Opt. 2011;31(2):123-36. 51. Prieto L, Lamarca R, Casado A. La evaluación de la fiabilidad en las observaciones clínicas: el coeficiente de correlacion intraclase. Med Clin. $1998 ; 110(4): 142-3$.

52. McAlinden C KJ, Pesudovs K. A comprehensive evaluation of the precision (repeatability and reproducibility) of the Oculus Pentacam HR. Invest Ophthalmol Vis Sci. 2011;29(52(10)):7731-7.

53. Gardiner SK, Johnson CA, Spry PG. Normal age-related sensitivity loss for a variety of visual functions throughout the visual field. Optom Vis Sci. 2006; 83 $(7): 438-443$.

54. Maddess, T. The Influence of Sampling Errors on Test-Retest Variability in Perimetry. Invest. Ophthalmol. Vis. Sci. 2012;52:1014-1022.

55. Govert P H, Ponsioen T L, Jansonius N M. Learning effect, normal range, and test-retest variability of Frequency Doubling Perimetry as a function of age, perimetric experience, and the presence or absence of glaucoma. Ophthal. Physiol. Opt. 2003;23:535-540. 
56. Brainard D. Cone contrast and opponent modulation colour spaces. In Kaiser PM, Boynton RMHuman Colour Vision Opatical Society of America Washington DC. 1996:563-79.

57. King-Smith PE, Carden D. Luminance and opponent-colour contributions to visual detection and adaptation and to temporal and spatial integration. J Opt Soc Am. 1976;66:709-717.

58. Morilla A, Anton A, Jimenez B, Rodriguez C, Martinez V, Fallon M, Capilla P, Luque MJ, Felipe A, Artigas JM. ATD perimetry in glaucoma and ocular hypertensive patients. A preliminar study. 10th Congress of the European Association for Vision and Eye Research (EVER 2007) pp-64 Abstract book. 


\section{FIGURE LEGENDS}

Figure 1: Results from the repeatability (within-observer) study for each of the 21 testing points in the visual field, for our four stimuli (A-Parvo, A-Magno, RG and BY). The visual field appears divided in $10^{\circ} \times 10^{\circ}$ regions, centered on each testing points, which are coded according to the ICC value or to the result of the Friedman test, as appropriate. ICC: excellent (dark gray), good (light gray). Friedman test: repeatable (white).

Figure 2: Results from the reproducibility (between-observer) study for the 21 testing points in the visual field, four our four stimuli (A-Parvo, A-Magno, RG and BY). The visual field appears divided in $10^{\circ} \times 10^{\circ}$ regions, centered on each testing points, which are coded according to the ICC value or to the result of the Friedman test, as appropriate. ICC: excellent (dark gray), good (light gray). Friedman test: reproducible (white). 

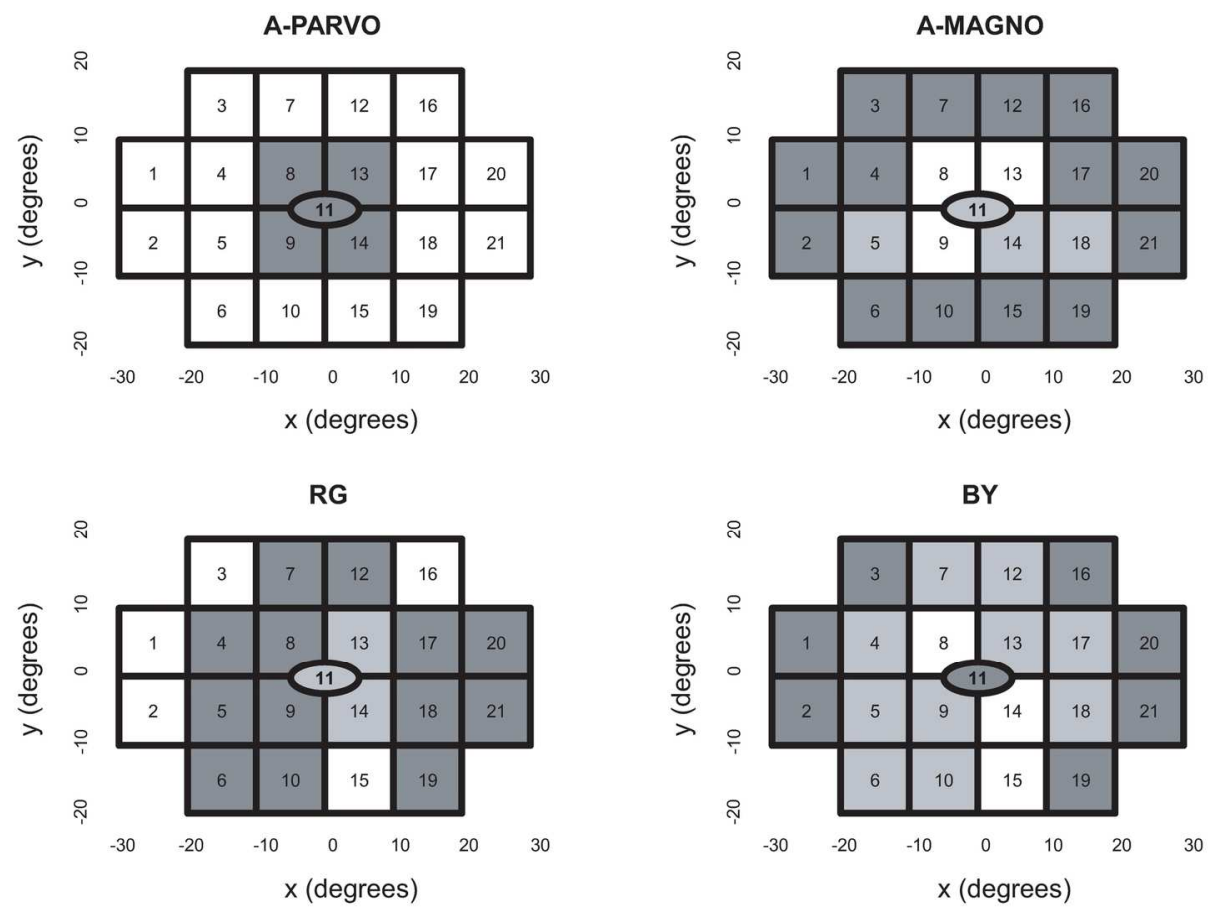

ICC: excelent

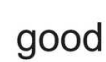

Friedman: repeatable

Results from the repeatability (within-observer) study for each of the 21 testing points in the visual field, for our four stimuli (A-Parvo, A-Magno, RG and BY). The visual field appears divided in $10^{\circ} \times 10^{\circ}$ regions, centered on each testing points, which are coded according to the ICC value or to the result of the Friedman test, as appropriate. ICC: excellent (dark gray), good (light gray). Friedman test: repeatable (white). $166 \times 132 \mathrm{~mm}(300 \times 300 \mathrm{DPI})$ 

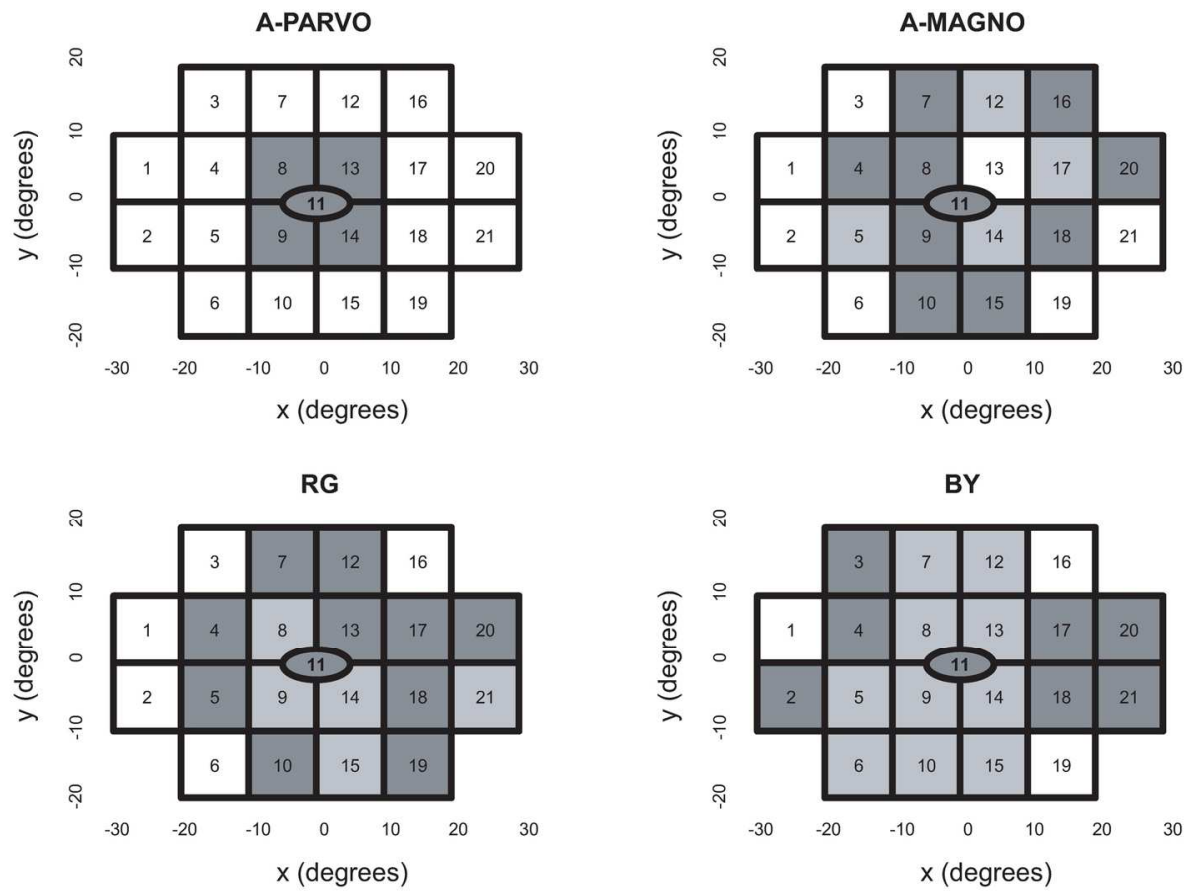

ICC: excelent

good

Friedman: reproducible

Results from the reproducibility (between-observer) study for the 21 testing points in the visual field, four our four stimuli (A-Parvo, A-Magno, RG and BY). The visual field appears divided in $10^{\circ} \times 10^{\circ}$ regions, centered on each testing points, which are coded according to the ICC value or to the result of the Friedman test, as appropriate. ICC: excellent (dark gray), good (light gray). Friedman test: reproducible (white). $166 \times 130 \mathrm{~mm}(300 \times 300$ DPI) 


\section{APPENDIX}

Stimuli were generated on a 17 inch LG Flatron F700P CRT monitor, configured to have a horizontal resolution of 1280 lines and a $72 \mathrm{~Hz}$ frame rate, driven by a 12-bits video controller (Bits++, from Cambridge Research Systems). The system was colorimetrically characterized and gamma corrected using the ColorCAL colorimeter and the Cambridge Research Systems Toolbox for MATLAB.

Observers are initially shown a spatially uniform achromatic field, with chromaticity coordinates $\mathrm{X}_{\mathrm{CIE}}=0.2709, \mathrm{y}_{\mathrm{CIE}}=0.2966$ and luminance equal to 45 $\mathrm{cd} / \mathrm{m}^{2}$, covering a $60^{\circ}$-horizontal by $40^{\circ}$-vertical fovea-centred area, and are asked to fixate a central $0.5^{\circ}$-wide black cross. The stimuli appearing on this background are flickering achromatic, red-green and blue-yellow gratings, with Gaussian smoothed borders. Testing points are arranged on a $4 \times 6$ regular grid -though the four corners of the grid are not tested-, with an additional point at the fovea (see Fig. A1a). Not considering the fovea, the grid spacing is $10^{\circ}$ and the offset from the vertical and horizontal meridians is $5^{\circ}$. The stimulus colour is defined by a vector whose components represent the changes in the responses, $R$, in the achromatic (A), red-green (RG) and blue-yellow (BY) mechanisms of the opponent modulation space ${ }^{31,32}$, computed using Brainard's formulation ${ }^{56}$. If $\Delta \vec{R}_{0}$ is the vector in this space defining the direction along which we are measuring the subject's threshold and the amplitude of the stimulus at a given trial and $\Delta \vec{R}(x, y, t)$ is the vector defining the stimulus at each spatial location $(x, y)$, measured (in degrees) from the testing point, at instant $t$ (in seconds) after stimulus onset, we have: 


$$
\Delta \vec{R}(x, y, t)=\Delta \vec{R}_{0} \cdot \sin \left(2 \pi f_{x} x+\frac{\pi}{2}\right) \cdot g(r) \sin 2 \pi f_{t} t \cdot h(t) \cdot \operatorname{rect}\left(\frac{x}{a}, \frac{y}{a}\right) E q \cdot A .1
$$

where $f_{x}$ and $f_{t}$ are the spatial and temporal frequencies of the stimulus, and " $a$ " is the angular size of the window containing the stimulus $\left(5^{\circ}\right)$. The functions $g(r)$ and $h(t)$ in Equation $A .1$ are, respectively, the spatial and the temporal envelope of the stimulus and are defined as follows:

$$
g(r)=\left\{\begin{array}{ccc}
1 & \text { if } & 0 \leq r \leq r_{0} \\
\exp \left\{-\frac{\left(r-r_{0}\right)^{2}}{2 \sigma^{2}}\right\} & \text { if } \quad r>r_{0}
\end{array}\right.
$$

Eq. A.2

$$
\begin{aligned}
& \text { where } r^{2}=x^{2}+y^{2}, r_{0}=1.5^{\circ} \text { and } \sigma=(1 / 3)^{\circ} ; \\
& \qquad h(t)=\left\{\begin{array}{c}
\exp \left\{-\frac{\left(t-t_{0}\right)^{2}}{2 \sigma_{t}^{2}}\right\} \quad \text { if } \quad 0 \leq t \leq t_{0} \\
11 \text { if } t_{0}<t \leq T_{s}-t_{0} \\
\exp \left\{-\frac{\left(t-T_{s}+t_{0}\right)^{2}}{2 \sigma_{t}^{2}}\right\} \quad \text { if } \quad T_{s}-t_{0}<t \leq T_{s}
\end{array}\right.
\end{aligned}
$$

Eq. A.3

where $T_{s}=1 \mathrm{~s}$ is the maximum presentation time, $t_{0}$ equals to $100 \mathrm{~ms}$ and $\sigma_{\mathrm{t}}=\mathrm{t}_{0} / 3$. These functions were introduced to smooth spatial-temporal transients that may constitute a cue for detection by an undesired mechanism. 
During a measurement session, the direction of vector $\Delta \vec{R}_{0}$ is fixed and coincides with one of the three cardinal directions of the space -that is, the direction isolating one of the mechanisms. In Figure A1 we show the limits and directions of the colour palettes in the CIE chromaticity diagram (Fig. A1b), and examples of the spatial and the temporal profiles (Fig. A1c-d), as well as a sample of stimuli in each of the cardinal directions (Fig. A1e-h).

\section{Insert Figure A1 here}

Stimuli are labelled as "Mechanism (A, RG, or BY)"-“Spatial Frequency (0.5 or 4 ) in cycles per degree (cpd)" /"Temporal Frequency (2 or 12) in Hertz $(\mathrm{Hz})$ ". To evaluate the achromatic mechanism, a stimulus favouring the magnocellular pathway $(\mathrm{A}-0.5 \mathrm{cpd} / 12 \mathrm{~Hz})$ and another one favouring the parvocellular pathway $(\mathrm{A}-4 \mathrm{cpd} / 2 \mathrm{~Hz})$ were chosen ${ }^{43}$. The red-green and blueyellow chromatic mechanisms, putatively mediated by the parvo and koniocellular pathways, respectively ${ }^{43}$, were evaluated with two stimuli of the same spatial and temporal frequency (RG-0.5cpd/2Hz and BY-0.5cpd/2Hz). The procedure described is similar to the one used by King-Smith for colour contrast thresholds, except for the spatial and temporal profile of the stimulus ${ }^{57}$.The stimuli used in this study were chosen after previous measurements covering the entire frequency range for each mechanism showed that the device had enough dynamic range to determine thresholds of subjects up to 70 years old ${ }^{44}$ and after measurements with pathological subjects suggested the possible utility of these stimuli in detection of functional damage $^{33,34,58}$. 


\section{FIGURE LEGEND}

Figure A1: (a) Array of testing points, (b) RG and BY cardinal directions in the colour space used in the experiment (CIE1931). The triangle represents the locus of colours generable by the monitor and the thick lines the maximum amplitude range available. (c) Spatial profile of a stimulus as shown in the CRT monitor. (d) Temporal profile of the stimulus. (e-h) Single frame of each of the four stimuli used in the experiment. 
a)

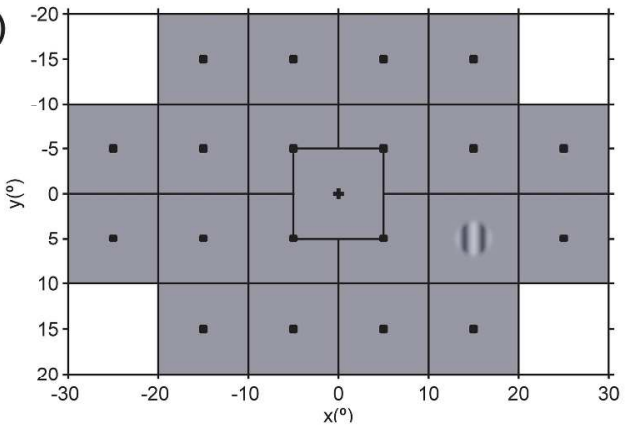

b)

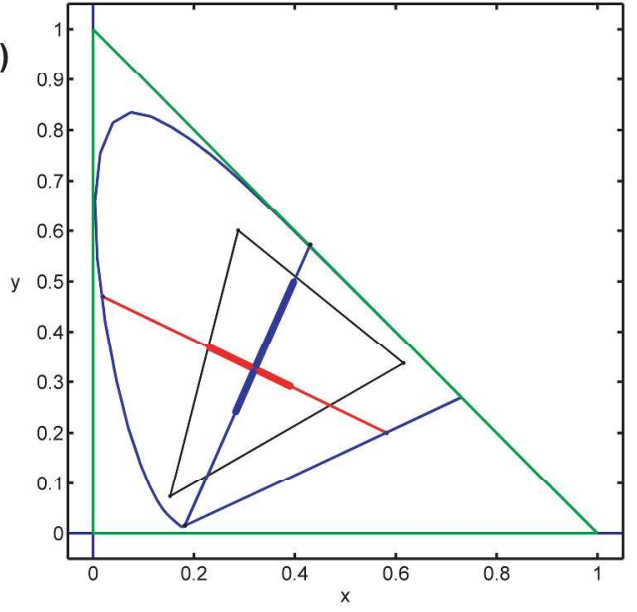

c)

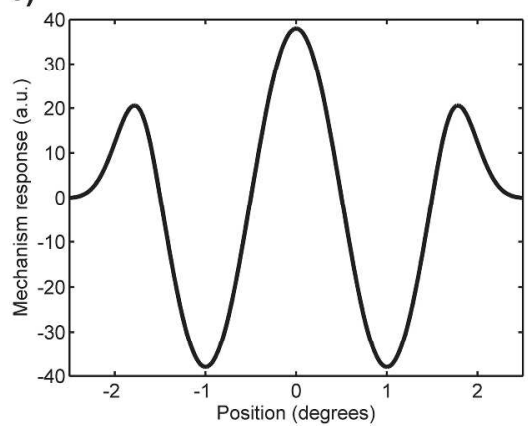

d)

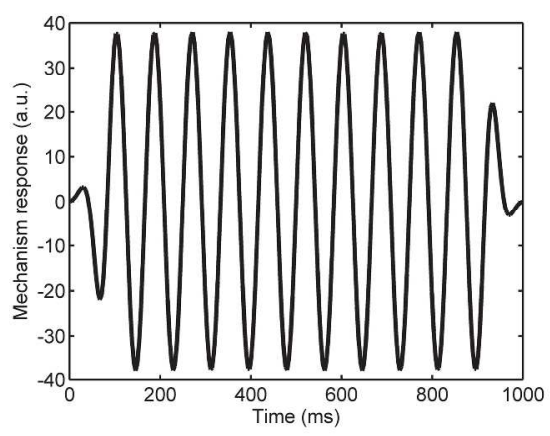

(a) Array of testing points, (b) RG and BY cardinal directions in the colour space used in the experiment, plotted in the CIE1931 color space. The triangle represents the locus of colours generable by the monitor and the thick lines the maximum amplitude range available. (c) Spatial profile of a stimulus as shown in the CRT monitor. (d) Temporal profile of the stimulus. $274 \times 227 \mathrm{~mm}(300 \times 300$ DPI) 
e)

f)

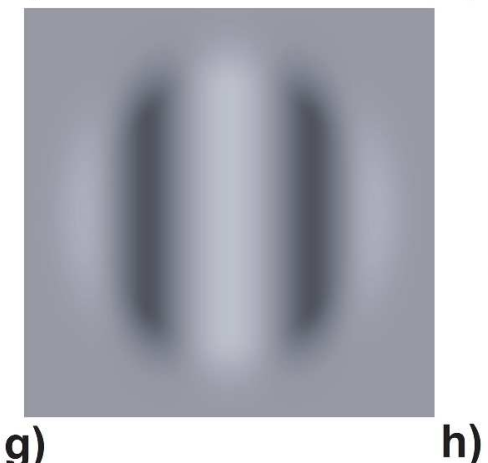

g)

h)
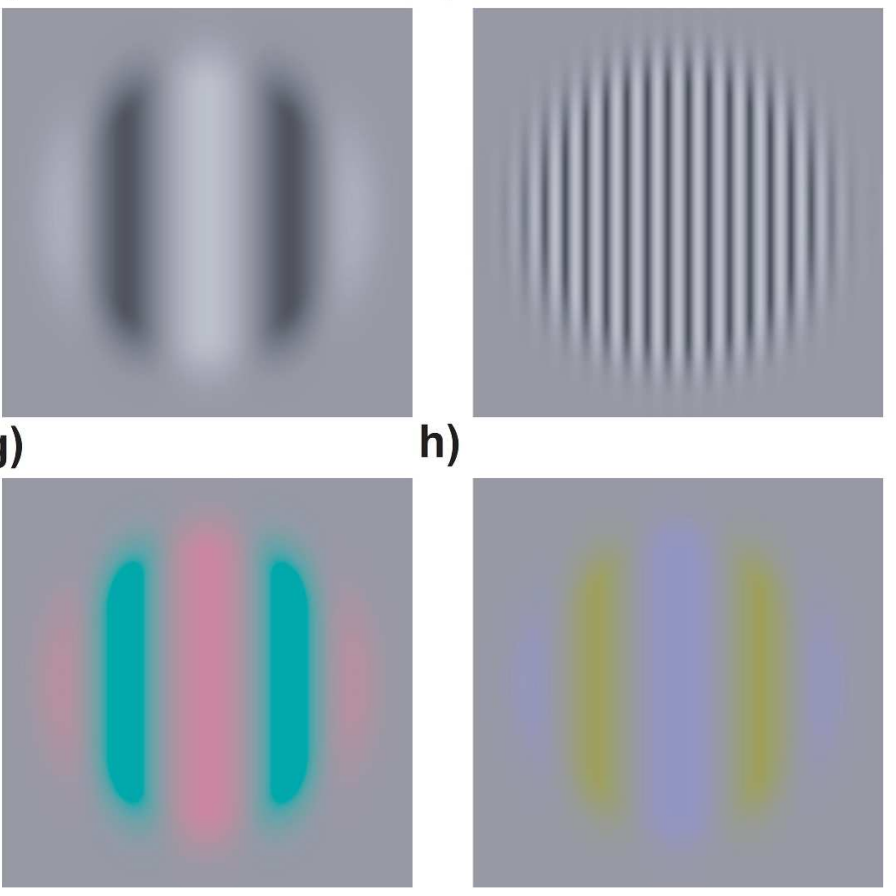

(e-h) Single frame of each of the four stimuli used in the experiment. $274 \times 455 \mathrm{~mm}(300 \times 300$ DPI) 